\title{
Interfacial water: A first principles molecular dynamics study of a nanoscale water film on salt
}

\author{
Li-Min Liu, ${ }^{1}$ Matthias Krack, ${ }^{2}$ and Angelos Michaelides ${ }^{1, a)}$ \\ ${ }^{1}$ Department of Chemistry and London Centre for Nanotechnology, University College London, \\ London WC1E 6BT, United Kingdom \\ ${ }^{2}$ Paul Scherrer Institut, CH-5232 Villigen-PSI, Switzerland
}

(Received 2 February 2009; accepted 20 May 2009; published online 17 June 2009)

\begin{abstract}
Density functional theory (DFT) molecular dynamics simulations of a thin $(\sim 15 \AA)$ water film on $\mathrm{NaCl}(001)$ have been performed, with the aim of understanding the structural and dynamic properties of this important interfacial water system. The interaction of the water film with the surface orders the water molecules in the immediate vicinity of the interface. This is reflected by oscillations in the planar-averaged water density distribution along the surface normal that extend to about $8 \AA$ from the surface. The interaction with the substrate leaves many of the water molecules in the immediate vicinity with broken hydrogen bonds and as a consequence considerably reduced dipole moments. Indeed a clear correlation between the number of hydrogen bonds which a water molecule is involved in and its dipole moment for both water on $\mathrm{NaCl}$ and bulk water is observed. How the DFT results obtained here compare to those obtained with various empirical potentials is briefly discussed. (C) 2009 American Institute of Physics. [DOI: 10.1063/1.3152845]
\end{abstract}

\section{INTRODUCTION}

At room temperature and at relative humidity below the deliquescence point of $75 \% \mathrm{NaCl}$ crystals are covered in a thin film of water believed to be about a nanometer thick or so. ${ }^{1,2}$ However, the properties of this interfacial water system, which essentially represents the "native" state of $\mathrm{NaCl}$ surfaces, remain unclear. Important basic questions, such as what the dynamic liquid structure, density variations, hydrogen bonding network, and electronic properties of the molecules in the adlayer are, remain unanswered. In the current article we address these and several other issues with the aid of $a b$ initio molecular dynamics (MD) simulations.

Considerable experimental and theoretical work has been devoted to the study of water- $\mathrm{NaCl}$ interfaces. Within the present context in which atomic and molecular-level insight is targeted, most of this work has been of the surface science style in which "frozen" water adsorption structures have been examined at cryogenic temperatures and under ultrahigh vacuum (UHV) conditions. Under these conditions two periodic water overlayers have been observed for the first wetting layer of water on $\mathrm{NaCl}(001)$ : $\mathrm{A} c(4 \times 2)$ and a $(1 \times 1)$ structure. $^{3,4}$ The circumstances under which each overlayer forms was somewhat controversially discussed until Toennies et $a l .{ }^{5}$ showed that exposure of the $(1 \times 1)$ layer to low energy electrons caused it to transform into the $c(4$ $\times 2$ ) overlayer. Both structures have been modeled with density functional theory (DFT) by a number of groups, ${ }^{6,7}$ with Cabrera-Sanfelix et al. ${ }^{7}$ concluding recently that the $(1 \times 1)$ and $c(4 \times 2)$ structures were essentially degenerate.

In the high coverage regime and under ambient conditions, the main focus of previous experimental studies has

\footnotetext{
a) Author to whom correspondence should be addressed. Electronic mail: angelos.michaelides@ucl.ac.uk.
}

been on exploring the onset of dissolution. ${ }^{8-12}$ Peters and Ewing $^{1}$ used infrared spectroscopy to establish that under ambient conditions, water adsorbs onto the surface of $\mathrm{NaCl}(001)$ into a liquidlike thin film. The film grew to an approximate thickness of 3 ML before the onset of deliquescence. Arsic et al. ${ }^{13}$ used surface x-ray diffraction to explore the water $/ \mathrm{NaCl}(001)$ interface at different relative humidities. It was suggested that at relative humidity above $45 \%$, the water molecules in the contact layer possess a considerable degree of lateral ordering. Although Arsic et al. did not expand upon this suggestion, as we will show below, it does appear to be consistent with the results of our first principles MD simulations.

On the theoretical side, there have been several empirical force field MD simulations of aqueous water films on $\mathrm{NaCl}$ surfaces. ${ }^{14,15}$ Anastasiou et al. ${ }^{14}$ first modeled a water film on perfect $\mathrm{NaCl}(001)$, finding variations in the planaraveraged water density along the surface normal, with a large peak in the planar-averaged water density at the immediate water $/ \mathrm{NaCl}$ interface. Shinto et al. ${ }^{16}$ studied the characteristics of a water film on perfect $\mathrm{NaCl}(001)$ and $\mathrm{NaCl}(011)$. Again they found buildups in the planar-averaged water density at the $\mathrm{NaCl}$ interfaces. Indeed at the (100) surface a planar-averaged water density maximum of $\sim 3.4 \mathrm{~g} / \mathrm{cm}^{3}$ was observed. Stöckelmann and Hentschke, ${ }^{17}$ using a polarizable water model, came to a similar conclusion about the planar-averaged water density maximum at the $\mathrm{NaCl}(001)$ interface, observing a density maximum of $\sim 2.7 \mathrm{~g} / \mathrm{cm}^{3}$. The fact that the planar-averaged density distribution exhibits variations along the surface normal indicates that the liquid near the surface is perturbed by the interface, with the molecules in the immediate interfacial layer expected the show more ordering than those in the bulk of the liquid. Indeed ordering of liquids at interfaces and the density oscil- 
TABLE I. Dependence of the adsorption energy $E_{\mathrm{ads}}$ and the distance between the $\mathrm{Na}$ and $\mathrm{O}$ of a water monomer on the basis set employed. For the first four data columns, $\mathrm{Na}$ and $\mathrm{Cl}$ are DZVP and TZV2P, respectively. For the last column, $\mathrm{Na}$ and $\mathrm{Cl}$ are TZV2P and QZV2P, respectively. For these calculations a three layer slab with a water coverage of $0.5 \mathrm{ML}$ is used. The basis set setup employed for the $a b$ initio MD simulations is indicated in bold.

\begin{tabular}{lcccccc}
\hline \hline \multirow{3}{*}{ Basis set } & $\mathrm{H}, \mathrm{O}$ & $\mathrm{DZVP}$ & TZVP & TZV2P & QZV2P & QZV2P \\
& $\mathrm{Na}$ & DZVP & DZVP & DZVP & DZVP & TZV2P \\
& $\mathrm{Cl}$ & TZV2P & TZV2P & TZV2P & TZV2P & QZV2P \\
$E_{\text {ads }}{ }^{\mathrm{a}}(\mathrm{eV})$ & & -0.603 & -0.481 & $\mathbf{- 0 . 4 6 4}$ & -0.420 & -0.419 \\
$\mathrm{Na}-\mathrm{O}(\AA)$ & & 2.418 & 2.426 & $\mathbf{2 . 4 2 7}$ & 2.430 & 2.429 \\
\hline \hline
\end{tabular}

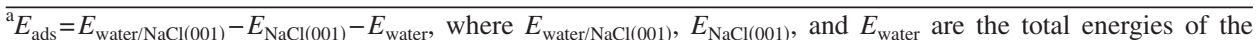
water/ $\mathrm{NaCl}(001)$ adsorption system, the clean $\mathrm{NaCl}(001)$ surface, and an isolated gas phase water molecule, respectively.

lations this implies is now a well-established feature of solidliquid interfaces, in general (see, e.g., Refs. 18-25). However, for water on $\mathrm{NaCl}(001)$, given the sensitivity of the results to the details of the force field interaction parameters employed, it is worthwhile to explore the liquid water $/ \mathrm{NaCl}$ interface with a predictive first principles approach. Previous first principles studies have focused on examining water in the bilayer or sub-bilayer regime and mostly they have been concerned with establishing low total energy structures at 0 $\mathrm{K}^{26-29}$ Mainly this is because exploring the properties of liquid-solid interfaces remains a challenge for first principles theory due to the often computationally prohibitive twin demands of large system sizes and long timescales. Indeed for these reasons there have only been a handful of such studies reported to date. ${ }^{25,30-33}$ There have also been a number of first principles studies dealing with the interaction of water with $\mathrm{NaCl}$ clusters which are likely to be of some relevance to $\mathrm{NaCl}$ dissolution, a topic that is beyond the scope of the current study. ${ }^{34-40}$

In an attempt to better understand the properties of the liquid water/salt interface, we present here $a b$ inito $\mathrm{MD}$ simulations of a nanometer thick water film on the perfect $\mathrm{NaCl}(001)$ surface. This is a full account of a previous communication. ${ }^{41}$ The properties we focus on and aim to shed light on are the planar-averaged water density profile, the interfacial structure of water molecules, the network of hydrogen bonds, and electronic structures of the molecules in the overlayer. To begin, we describe the details of the computational setup employed (Sec. II). Following this the properties of the water $/ \mathrm{NaCl}$ interface obtained from our $\mathrm{MD}$ simulations are analyzed (Sec. III). The dependence of the planar-averaged water density profile on temperature is then briefly considered and the density profile obtained from our first principles simulations is compared to those obtained with a few simple empirical potential parametrizations (Sec. IV). We close with a short summary and some conclusions (Sec. V).

\section{METHOD}

The DFT calculations reported here have been performed with the CP2K/QUICKSTEP (Ref. 42) program, which uses a hybrid Gaussian and planewave basis set and is based on DFT. The Perdew-Burke-Ernzerhof ${ }^{43}$ (PBE) exchangecorrelation functional has been used along with norm con- serving Goedecker-Teter-Hutter ${ }^{44}$ pseudopotentials. The well-known problem of nonlinear core-valence electron exchange and correlation for $\mathrm{Na}$ (Ref. 45) has been eliminated in the present study by treating the $\mathrm{Na} 2 s$ and $2 p$ electrons as valence electrons. The wave functions of the valence electrons are expanded in terms of Gaussian functions with a triple- $\zeta$ doubly polarized basis set (TZV2P) for $\mathrm{H}, \mathrm{O}$, and $\mathrm{Cl}$, and a double- $\zeta$ single polarized basis set (DZVP) for Na. For the auxiliary basis set of plane waves, a 320 Ry density cutoff is used. The quality of this basis set has been fully checked. As shown in Table I, for example, compared with a considerably larger combination of QZV2P and TZV2P basis sets, the adsorption energy of a water monomer on $\mathrm{NaCl}$ changes by $45 \mathrm{meV}$ and the Na-O distance by only $0.002 \AA$.

For the water $/ \mathrm{NaCl}$ system, a $p(3 \times 4) \mathrm{NaCl}(001)$ supercell is used, containing a three layer $\mathrm{NaCl}$ slab and 89 water molecules, as shown in Fig. 1(a). A computed lattice constant for $\mathrm{NaCl}$ of $5.72 \AA$ has been used, which is within $2 \%$ of experimental value of $5.63 \AA^{46}{ }^{46}$ The adsorbed water layer is about $15 \AA$ thick and there is a further $25 \AA$ vacuum used to

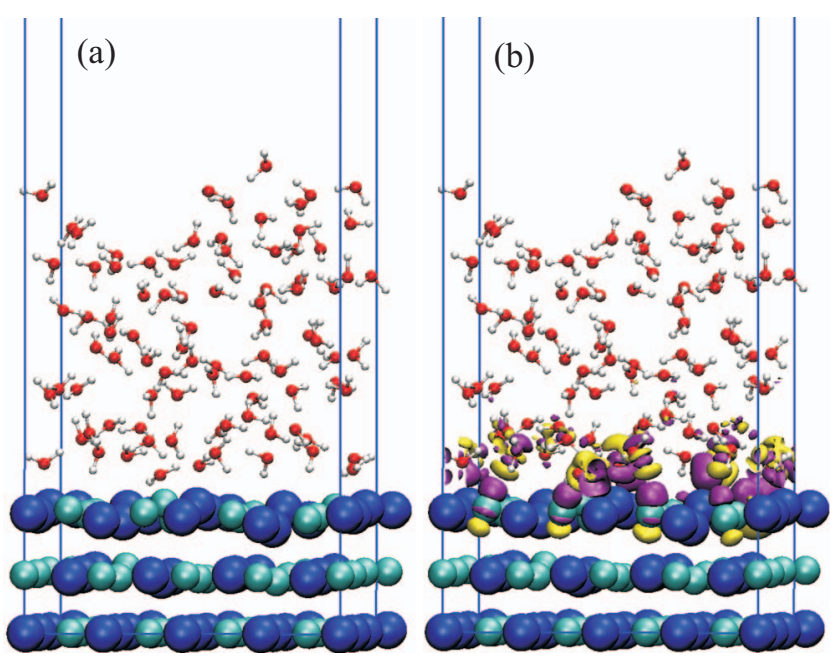

FIG. 1. (Color) (a) Part of the water $/ \mathrm{NaCl}$ simulation supercell at one particular snapshot of the MD simulation. (b) Isosurface of electron density rearrangement $\Delta \rho^{e}$ [as obtained from Eq. (1)] at the same snapshot of the simulation. A constant density isosurface of $\pm 0.007 e / \AA^{3}$ is displayed, with yellow indicating positive regions (electron accumulation) and purple negative regions (electron depletion). The $z$ axis is the long axis of the simulation cell. Red, white, dark blue, and light blue spheres represent $\mathrm{O}, \mathrm{H}, \mathrm{Na}$, and $\mathrm{Cl}$ atoms, respectively. 
TABLE II. Dependence of the adsorption energy $E_{\text {ads }}$ and the distance between the $\mathrm{Na}$ and $\mathrm{O}$ of a water monomer on an $\mathrm{NaCl}(001)$ surface $(0.5 \mathrm{ML})$ for different numbers of $\mathrm{NaCl}$ layers. A DZVP basis set is used for $\mathrm{Na}$, TZV2P for $\mathrm{Cl}, \mathrm{H}$, and $\mathrm{O}$.

\begin{tabular}{lrrrrr}
\hline \hline NaCl layers & 2 & 3 & 4 & 5 & \multicolumn{1}{c}{6} \\
\hline$E_{\text {ads }}(\mathrm{eV})$ & -0.460 & -0.464 & -0.453 & -0.449 & -0.455 \\
$\mathrm{Na}-\mathrm{O}(\AA)$ & 2.475 & 2.427 & 2.428 & 2.429 & 2.429 \\
\hline \hline
\end{tabular}

separate slabs in adjacent cells. A three layer $\mathrm{NaCl}$ slab was selected after a series of tests, which established that the adsorption energy of a water monomer on $\mathrm{NaCl}(001)$ is rather insensitive to the number of layers used in the calculations. For example, as can be seen from Table II, the monomer adsorption energy and structure are within $10 \mathrm{meV}$ and $0.002 \AA$ of that obtained on a six layer thick slab.

The liquid water $/ \mathrm{NaCl}$ simulations began with a $\mathrm{MD}$ simulation performed with empirical potentials $(200 \mathrm{ps} d u-$ ration at $\sim 300 \mathrm{~K})$. For these simulations the Born-MayerHuggins potential $^{47}$ was used for $\mathrm{NaCl}$, TIP3P (Ref. 48) for water, and the water-ion interaction parameters were taken from Ref. 49 (Table III). The final structure obtained from the empirical potential simulations was then used as the initial configuration for the first principles MD simulations, which were of the Born-Oppenheimer (BO) type within the microcanonical $(N V E)$ ensemble. Although the phase diagram of PBE water has not yet been determined, previous studies have shown that at room temperature DFT-PBE water is overstructured and has a diffusion coefficient much smaller than experiment. ${ }^{25,50-53}$ To avoid this problem DFTPBE simulations of liquid water are often performed at high temperatures $(330-400 \mathrm{~K}){ }^{25,50-53}$ Here we set a target temperature of $330 \mathrm{~K}$ for the $N V E$ simulations. For comparison, a simulation has also been performed at a target temperature of $360 \mathrm{~K}$, which we discuss in Sec. IV. The first principles simulation of the as-described water film on $\mathrm{NaCl}(001)$ was run for a total of $35 \mathrm{ps}$. For the first 5 ps velocity rescaling was performed. The nuclear equations of motion have been integrated using a standard velocity Verlet algorithm with a $1.0 \mathrm{fs}$ time step and deuterium masses for the hydrogen atoms used in order to facilitate a larger timestep, although we will refer to water as $\mathrm{H}_{2} \mathrm{O}$ throughout. By using D masses for the hydrogen atoms the nuclear quantum effects of water are also reduced. ${ }^{53}$ Only the final 20 ps of the simulation was used for analysis and data acquisition. During the MD simulation, a strict convergence criterion for the electronic gradient, $10^{-7}$, is employed. This ensures stable microcanonical dynamics as illustrated by the plot of the total and potential energy of the system (Fig. 2). The measured total energy drift is smaller than $10^{-5} \mathrm{eV} / \mathrm{ps} /$ atom and the potential energy rather flat, especially the last 20 ps of the simulation. In addition we note that throughout the entire simulation, no $\mathrm{Na}$ or $\mathrm{Cl}$ ions dissolve into the water film nor do any water molecules dissociate within or desorb from the liquid water film.

BO MD simulations for a bulk liquid water reference system were also performed. This system was comprised of $32 \mathrm{D}_{2} \mathrm{O}$ molecules in a $9.853 \AA$ cubic cell (equivalent to a water density of $1 \mathrm{~g} / \mathrm{cm}^{3}$ ) and was examined with the same exchange-correlation functional, basis sets, ensemble and temperature, time step, etc., as the water $/ \mathrm{NaCl}$ calculations.

\section{RESULTS}

We now discuss the main results to come from our ab initio MD simulations. First, we discuss structural prop-

TABLE III. Force field parameters describing the water-ion interactions tested in this study. The parameters from Smith et al. were used to generate the initial structure that was used in the DFT-PBE simulations. $\sigma$ and $\varepsilon$ have their standard meanings and standard arithmetic $\left[\sigma_{i j}=\frac{1}{2}\left(\sigma_{i}+\sigma_{j}\right), \varepsilon_{i j}=\sqrt{\varepsilon_{i} \varepsilon_{j}}\right]$ combination rules were used to obtain the water-ion interaction parameters.

\begin{tabular}{|c|c|c|c|c|c|}
\hline & & \multicolumn{2}{|c|}{$\mathrm{Na}^{+}$} & \multicolumn{2}{|c|}{$\mathrm{Cl}^{-}$} \\
\hline & & $\begin{array}{c}\sigma \\
(\AA)\end{array}$ & $\begin{array}{c}\varepsilon \\
(\mathrm{kcal} / \mathrm{mol})\end{array}$ & $\begin{array}{c}\sigma \\
(\AA)\end{array}$ & $\begin{array}{c}\varepsilon \\
(\mathrm{kcal} / \mathrm{mol})\end{array}$ \\
\hline \multirow{2}{*}{ Smith et al. ${ }^{\text {a }}$} & $\mathrm{O}$ & 2.758 & 0.142 & 3.783 & 0.125 \\
\hline & $\mathrm{H}$ & $\ldots$ & $\ldots$ & $\ldots$ & $\ldots$ \\
\hline \multirow{2}{*}{ Hummer et al. ${ }^{\mathrm{b}}$} & $\mathrm{O}$ & 2.85 & 0.048 & 3.75 & 0.129 \\
\hline & $\mathrm{H}$ & $\cdots$ & $\ldots$ & $\cdots$ & $\ldots$ \\
\hline \multirow{2}{*}{ Pettitt et al. ${ }^{\mathrm{c}}$} & $\mathrm{O}$ & 2.72 & 0.134 & 3.55 & 0.134 \\
\hline & $\mathrm{H}$ & 1.31 & 0.360 & 2.14 & 0.360 \\
\hline \multirow{2}{*}{ Lynden-Bell et al. ${ }^{\mathrm{d}}$} & $\mathrm{O}$ & 2.876 & 0.125 & 3.785 & 0.125 \\
\hline & $\mathrm{H}$ & $\ldots$ & $\ldots$ & $\ldots$ & $\ldots$ \\
\hline
\end{tabular}

${ }^{2}$ Reference 49 .

${ }^{\mathrm{b}}$ Reference 80.

${ }^{\mathrm{c}}$ Reference 82.

${ }^{\mathrm{d}}$ Reference 81. 


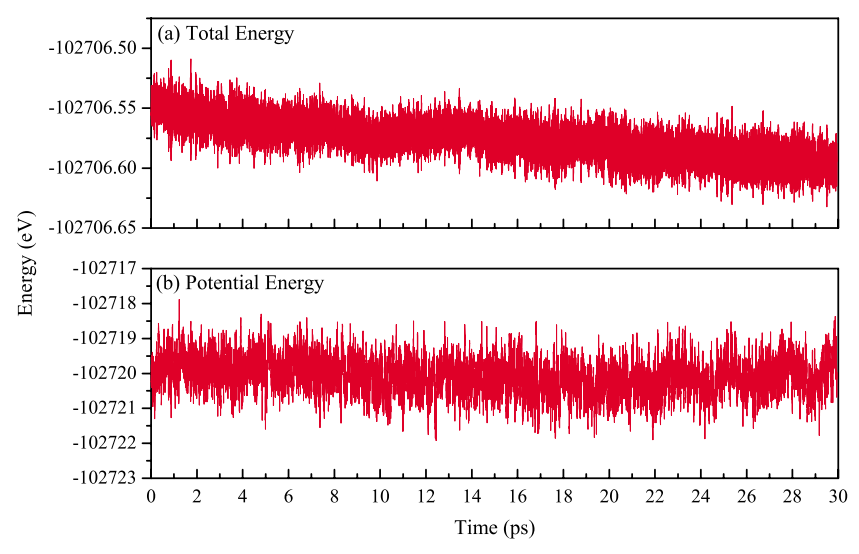

FIG. 2. (Color online) Evolution of the total energy (a) and potential energy (b) at $330 \mathrm{~K}$ for the water/NaCl system computed with DFT-PBE.

erties of the liquid overlayer, such as the density of water molecules along the surface normal, their orientational distribution, radial distribution functions (RDFs), and hydrogen bonding network. Following this, we study the interface structure in the immediate water $/ \mathrm{NaCl}$ contact layer. Certain electronic properties of the water film are reported in Sec. III F.

\section{A. Density profile of the water}

The first and probably most important property of the water film that we now discuss is the density distribution of water molecules. To this end the planar-averaged water density as a function of distance from the interface is shown in Fig. 3(a). The density profile depicted by the solid line in Fig. 3(a) (and the others reported in Figs. 11-13) was obtained by averaging the density within $0.125 \AA$ intervals along the $z$ axis. Tests of bins with other widths (0.063-0.25 $\AA$ ) demonstrated that the density profiles obtained are not particularly sensitive to this choice. For example, the dotted line in Fig. 3(a) was obtained by averaging the density along the $z$ axis within $0.063 \AA$ intervals, and it is practically indistinguishable from the one obtained with the $0.125 \AA$ bin. Large density oscillations are observed in the film. Such os- cillations are expected and a characteristic feature of water at an interface. ${ }^{18-25}$ The detailed nature of the oscillations in the density profile is specific to water on $\mathrm{NaCl}(001)$, which is what we examine now.

For discussion purposes, we consider the water film as being split into five separate layers as shown in Fig. 3(a). The division into layers is guided by the observed oscillations in the density profile, with the boundaries placed at minima on the density profile, except for the boundary between the fourth and fifth layers, which has been placed just before the density begins to drop to zero as the vacuum is approached. In the immediate vicinity of the interface, there are two intense peaks in the planar-averaged density (layers 1 and 2). The first peak within layer 1 has a density maximum $\sim 40 \%$ higher than that of equilibrium room temperature water, i.e., the density maximum is $1.4 \mathrm{~g} / \mathrm{cm}^{3}$. Water molecules in this region can be expected to be greatly overstructured compared with ideal bulk liquid water under the same conditions [although much less structured than in bulk ice, cf. the dashed line in Fig. 3(a)]. Later, upon consideration of the distribution of $\mathrm{O}$ and $\mathrm{H}$ atom densities we will show that this is indeed the case. For the second peak, corresponding to layer 2, the planar-averaged density is again rather high, $1.26 \mathrm{~g} / \mathrm{cm}^{3}$. Outside the second peak, there is a deep valley, where the density drops to only $0.3 \mathrm{~g} / \mathrm{cm}^{3}$. Quite few oxygen atoms are located at the boundary between layers 2 and 3, as can be seen from Fig. 3(b), which shows the specific planar-averaged densities associated with the $\mathrm{O}$ and $\mathrm{H}$ atoms. Only at a distance of $\sim 10 \AA$ from the surface does the density settle at the bulk liquid water value of $1 \mathrm{~g} / \mathrm{cm}^{3}$. We note that the equilibrium density of PBE liquid water may not be exactly $1 \mathrm{~g} / \mathrm{cm}^{3} .^{54,55}$ Thus the precise magnitude of the planar-averaged density oscillations observed here relative to PBE bulk liquid water is somewhat unclear. Because the water film is only about $15 \AA$ thick, the region with bulk liquid water density is narrow (3-4 $\AA$ ). Beyond this (layer 5) the density decreases to zero as the water/vacuum interface is approached, in a similar fashion to the previous DFT report of the liquid water/vacuum interface. $^{56}$

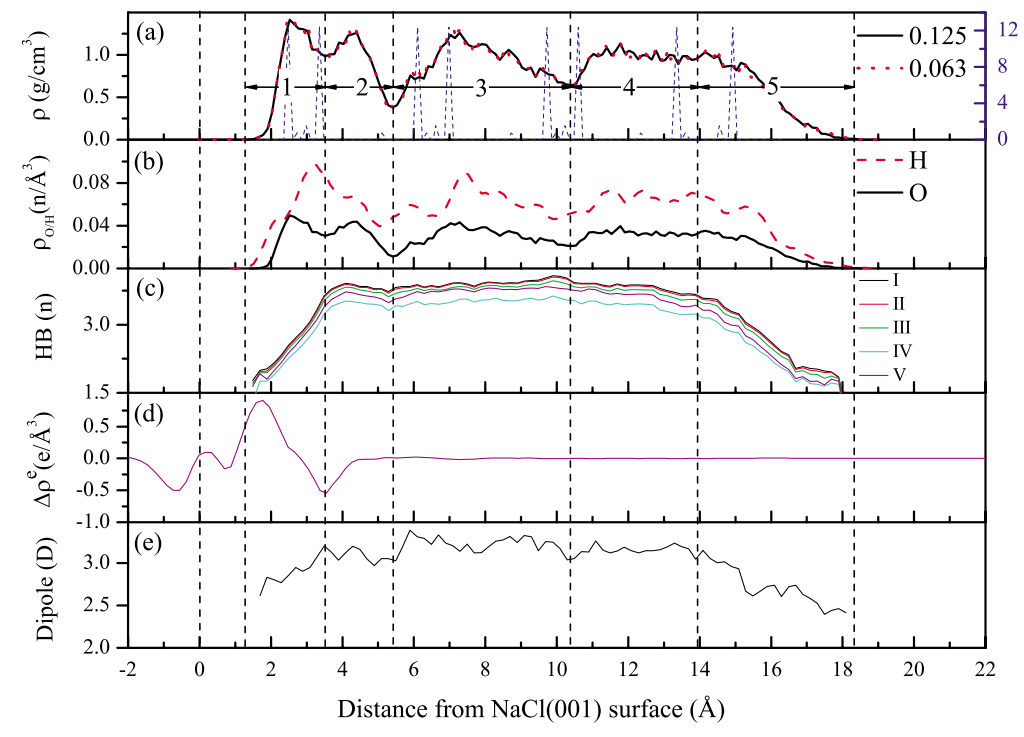

FIG. 3. (Color online) Selected properties of a liquid water film on $\mathrm{NaCl}(001)$ obtained from a DFT-PBE simulation at $330 \mathrm{~K}$. (a) Planar-averaged water density $(\rho)$ obtained by averaging the water density within $0.125 \AA$ (solid line) and $0.063 \AA$ (dotted line) intervals, along the $z$ axis. For comparison, the density of bulk ice Ih along the $z$ axis, obtained with $0.125 \AA$ intervals (dashed line), is also shown; (b) The planar-averaged density of $\mathrm{H}\left(\rho_{\mathrm{H}}\right)$ and $\mathrm{O}\left(\rho_{\mathrm{O}}\right)$ atoms. (c) Total number of hydrogen bonds per molecule for five different hydrogen bond definitions. (d) Electronic charge density difference $\left(\Delta \rho^{e}\right)$ between the water and $\mathrm{NaCl}$ surface. (e) Distribution of dipole moments per water molecule. The dashed vertical lines and numbers in (a) specify the layers into which the film is divided for analysis purposes. The average height of the first layer of the $\mathrm{NaCl}(001)$ is defined as the zero of the abscissa. 


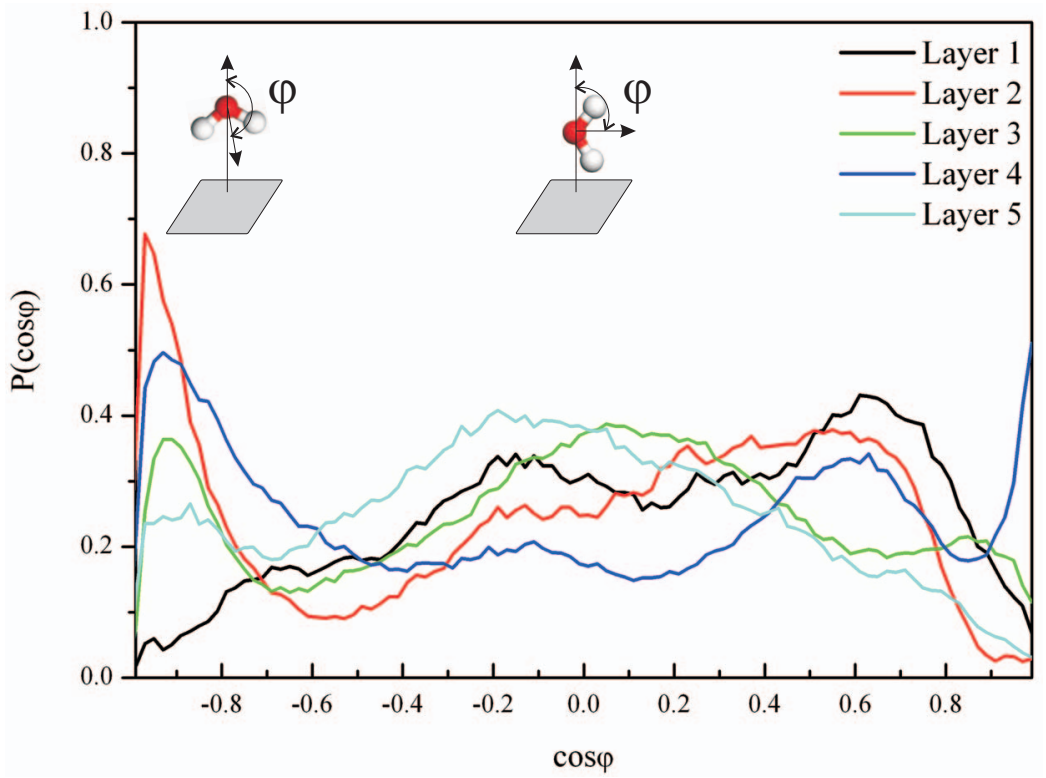

FIG. 4. (Color) Distribution of dipole moment orientations within each layer of the water film, as obtained from a DFT-PBE simulation at $330 \mathrm{~K} . \varphi$ is defined as the angle between the dipole vector of the water molecule and the surface normal.
The distributions of $\mathrm{O}$ and $\mathrm{H}$ atom densities within the film reveal further information about the overlayer [Fig. 3(b)]. First the $\mathrm{O}$ and $\mathrm{H}$ number density distributions differ significantly, implying again that there is appreciable order of the water molecules within the overlayer. Specifically, at the water $/ \mathrm{NaCl}$ interface, the hydrogen atoms are closer to the interface than the $\mathrm{O}$ atoms. Such results indicate that some of the water molecules have $\mathrm{OH}$ bonds which are directed at the interface. Second, the peaks and valleys for the separate $\mathrm{O}$ and $\mathrm{H}$ distributions do not exactly overlap. Indeed the position of the first peak of the $\mathrm{H}$ distribution is quite close to that of the first valley of the $\mathrm{O}$ (and water) density. Likewise there is a surplus of $\mathrm{H}$ atoms in the valleys in the $\mathrm{O}$ density separating layers 2 and 3 . Thus the $\mathrm{H}$ atoms play a role in connecting the densities of layers 1-3. After layer 3, the distributions of $\mathrm{H}$ and $\mathrm{O}$ atoms become quite flat, representative of a more inhomogeneous liquidlike overlayer. Finally, at the water/vacuum interface the $\mathrm{H}$ atom density extends about $0.5 \AA$ beyond that of the $\mathrm{O}$ atom density, indicating that a proportion of the $\mathrm{OH}$ bonds at this interface "dangles" into the vacuum.

\section{B. Water orientational distribution}

From the $\mathrm{O}$ and $\mathrm{H}$ planar-averaged density distributions, we have obtained qualitative insight into the orientations of the water molecules within the film. To obtain a more detailed picture the distribution of water dipole moment orientations within each layer has been examined. The dipole moment orientation $\varphi$ is defined as the angle between the dipole vector of the water molecule and the surface normal $(z$ axis of the unit cell). A homogeneous orientational distribution of water molecules within a layer would be represented by a weak or no dependence on $\cos \varphi$. A predominance of molecules in a layer with dipole moments directed away from the $\mathrm{NaCl}$ surface would result in a peak at $\cos \varphi>0$, and a predominance of molecules with their dipole moment oriented toward the $\mathrm{NaCl}$ surface would lead to a peak at $\cos \varphi<0$.
The calculated orientational distribution of molecular dipole moments is presented in Fig. 4. In layer 1, two main peaks are obtained. The first peak, $\cos \varphi=0.7$, corresponds to water molecules adsorbed on the surface with both of their $\mathrm{OH}$ bonds directed slightly away from the surface. Later (Sec. III E) we will show that these are associated with water molecules adsorbed above the Na sites of the substrate. The second smaller peak in the first layer at $\cos \varphi=0$ corresponds to molecules with their dipole moment almost parallel to the surface. Again, we will show below that this peak is associated with molecules close to $\mathrm{Cl}$ sites of the substrate with one of their $\mathrm{OH}$ bonds interacting with Cls. The orientational distribution within layer 2 resembles layer 1 to some extent but in addition has a large peak at $\cos \varphi=-0.95$, indicating the presence of a fraction of water molecules with their dipole directed at the $\mathrm{NaCl}$ surface. Layers 3 and 4 exhibit somewhat bimodal distributions with the former possessing large fractions of flat and downpointing molecules and the later large fractions of upright and downpointing molecules with relatively few aligned parallel to the surface. For the outermost layer, layer 5, a single broad maximum around $\cos \varphi=0$ is observed, indicating that most of the molecules at the water/vacuum interface are aligned approximately parallel to this interface.

\section{RDFs}

RDFs are a traditional means with which to explore the structural properties of liquids. Here, we examine the oxygen-oxygen $(\mathrm{O}-\mathrm{O})$ and oxygen-hydrogen $(\mathrm{O}-\mathrm{H})$ RDFs for each layer within the film. These are reported in Fig. 5 along with the calculated RDFs for bulk water. The bulk liquid water RDFs are in good agreement with previous DFT-PBE results with the first peak in the O-O RDF having a value of $g_{\mathrm{OO}} \approx 3.5$ at an $\mathrm{O}-\mathrm{O}$ separation of $2.7 \AA^{50,57} \mathrm{Al}-$ though the first peaks in the $\mathrm{O}-\mathrm{O}$ RDFs for the interfacial layers (layers 1 and 5) are at the same location $(2.7 \AA)$ as in the bulk, their heights are considerably reduced $\left(g_{\mathrm{OO}} \approx 2.2\right.$ for layers 1 and 5 compared to $g_{\mathrm{OO}} \approx 3.5$ for bulk). Likewise, 

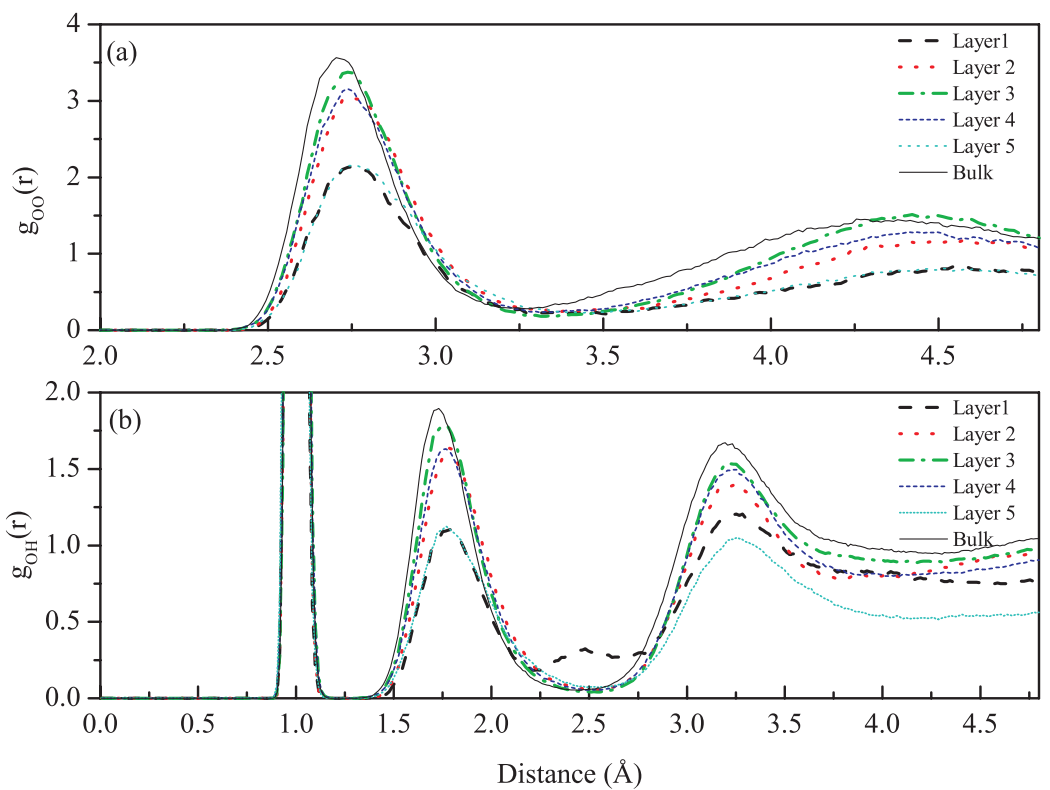

FIG. 5. (Color) Oxygen-oxygen (a) and oxygenhydrogen (b) RDFs for each layer within the water film on $\mathrm{NaCl}$ and bulk water, as obtained from DFT-PBE simulations at $330 \mathrm{~K}$. the height of the first $\mathrm{O}-\mathrm{H}$ intermolecular peak at $1.7 \AA$ is considerably reduced compared to bulk at the two interfaces. Specifically $g_{\mathrm{OH}}$ for layers 1 and 5 is $\sim 1.1$, compared to $g_{\mathrm{OH}} \sim 1.9$ for bulk water. These reductions in the peak heights in the RDFs are to be expected and simply a result of the reduced coordination brought about by the presence of the $\mathrm{NaCl}$ and vacuum interfaces. The preferred orientational distributions and high density of water molecules in layer 1 give rise to some changes in the $\mathrm{O}-\mathrm{H}$ intermolecular region: The O-H peak position is shifted slightly $(\sim 0.04 \AA)$ to longer distances and there is a new small peak at $\sim 2.5 \AA$. Moving into the interior of the water film, the first peak maxima of both the $g_{\mathrm{OO}}$ and $g_{\mathrm{OH}}$ recover quickly: Layers 2, 3 , and 4 have O-O RDF maxima of 3.02, 3.37, and 3.13, respectively.

\section{Network of hydrogen bonds}

Many of the properties of water are related to its network of hydrogen bonds. It is therefore worth considering how the hydrogen bonding properties of the thin supported water film examined here differ from those of bulk water. To this end, we computed the average number of hydrogen bonds the molecules in each layer are involved in. The answer to this question is, of course, sensitive to the definition of the hydrogen bond used, and there is no shortage of definitions of hydrogen bonds or debates as to the average number of hydrogen bonds in liquid water. ${ }^{50-53,56-63}$ The results obtained from five specific definitions (described in Ref. 61), plotted as a function of the distance from the interface, are shown in Fig. 3(c). It can be seen that all definitions yield qualitatively similar results, with only rather modest quantitative differences obtained from the five definitions. The main conclusion of this analysis is that there is a pronounced reduction in the average number of hydrogen bonds at the interfaces (water/ $\mathrm{NaCl}$ and water/vacuum), as expected.

Let us now consider the results of the hydrogen bond analysis in more detail, focusing specifically on the results obtained from definitions I and IV which predict the largest and smallest numbers of hydrogen bonds, respectively (Fig. 6 ). For bulk water, our results show that $76 \%$ (50\%) of the water molecules have four hydrogen bonds using definition I (IV). Such results for definition I agree well with previous DFT calculations. ${ }^{51,62,63}$ Moving to the water $/ \mathrm{NaCl}$ interface, most of water molecules in layer 1 have three hydrogen bonds ( $57 \%$ and $44 \%$ with definitions I and IV), only $6 \%$ $(4 \%)$ of the molecules have four hydrogen bonds, $33 \%$ $(40 \%)$ of the molecules have two hydrogen bonds, and only $3 \%(10 \%)$ have a single hydrogen bond for definition I (IV). This indicates that in terms of hydrogen bonds the water molecules close to the $\mathrm{NaCl}$ surface are undercoordinated. With increasing distance from the $\mathrm{H}_{2} \mathrm{O} / \mathrm{NaCl}$ interface, the network of hydrogen bonds recovers quickly. In fact, it can be seen from Fig. 6 that the hydrogen bonding distribution in layers 2-4 resembles that of bulk water rather closely. Differences from the bulk in terms of the proportion of molecules with various numbers of hydrogen bonds are never more than 5\%-10\% with both definitions I and IV. As for

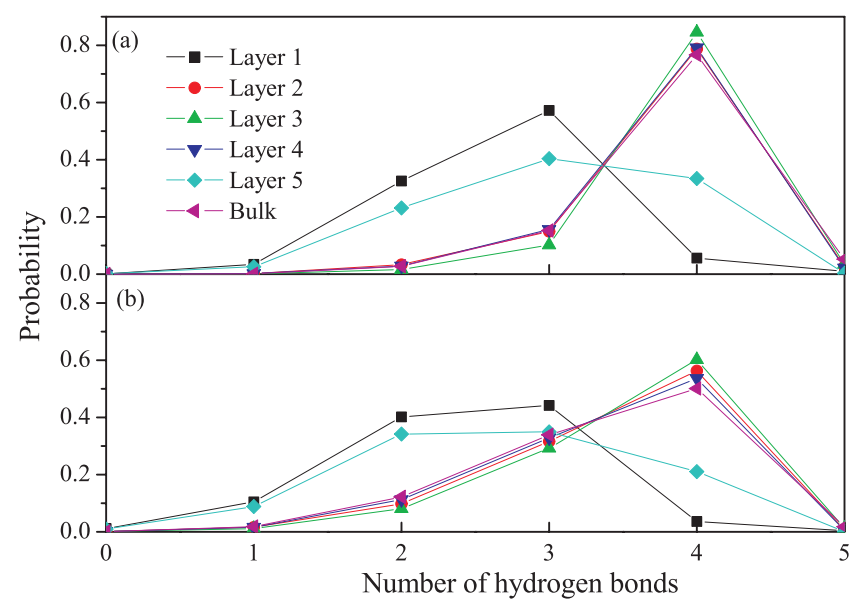

FIG. 6. (Color) Distribution of water molecules with different numbers of hydrogen bonds using definition I (a) and definition IV (b) for the numbers of $\mathrm{H}$ bonds between water molecules. See Ref. 61 for a description of definitions of I and IV. 


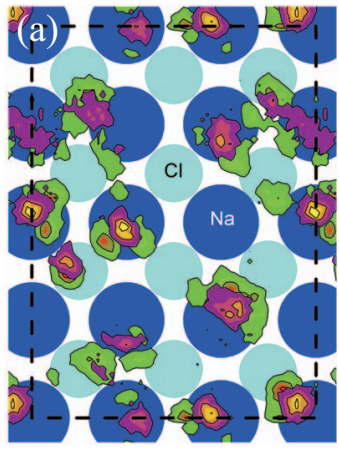

(c)

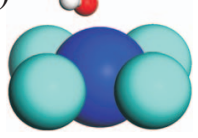

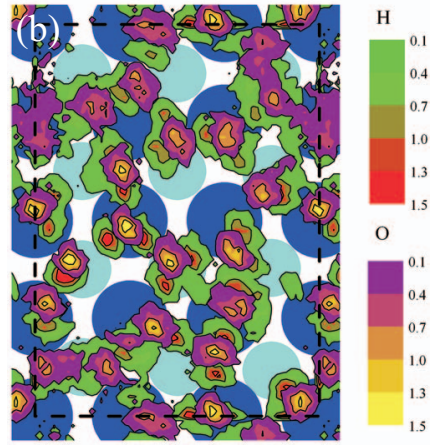

(d)

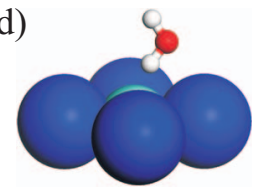

FIG. 7. (Color) (a) Spatial distribution functions of $\mathrm{O}$ and $\mathrm{H}$ atoms projected onto the $\mathrm{NaCl}(001)$ surface within layer 1 (a) and within layer 1 plus layer 2 (b). Two common adsorption geometries within layer 1 are also shown: (c) water above a $\mathrm{Na}$ ion and (d) water above a $\mathrm{Cl}$ ion.

layer 5, there are again quite a lot broken hydrogen bonds: Large proportions of the molecules are either threefold or twofold coordinated with only $40 \%(21 \%)$ of the molecules fourfold coordinated with definition I (IV). Thus, overall it is clear that the presence of each interface has a big impact on the hydrogen bonding network. However, the influence is rather short ranged, and once one is beyond about $4 \AA$ from each interface, the hydrogen bonding network largely resembles that of bulk water.

\section{E. The interfacial structure of water/ $\mathrm{NaCl}$}

We now focus on the details of the water structure within the first layer, aiming to understand how the water molecules interact with the $\mathrm{NaCl}$ surface. To this end we plot in Fig. 7(a) the spatial probability distribution of the oxygen and hydrogen atoms within the first layer projected onto the $\mathrm{NaCl}$ surface. This reveals that there are two typical adsorption structures for water molecules in the first layer. In one, the $\mathrm{O}$ atoms of the water molecules are located above Na sites [Fig. $7(\mathrm{c})]$ with the molecular dipole almost parallel to the surface. In the other a single $\mathrm{OH}$ bond of an upright water interacts with $\mathrm{Cl}$ ions [Fig. 7(d)]. We note that the adsorption structure at the $\mathrm{Na}$ site resembles the $1 \mathrm{ML}$ UHV structure of water on $\mathrm{NaCl}(001)$ suggested on the basis of helium atom scattering ${ }^{4}$ and the structure predicted by DFT for water monomer adsorption on $\mathrm{NaCl} .^{26-29,64}$ Throughout the entire trajectory we estimate that each adsorption configuration occurs with equal probability. On average, in a unit cell there are about 11 water molecules adsorbed on the surface within layer 1 (the average oxygen atom number within layer 1 for the whole trajectory is 11.05 with a standard deviation of 1.5 ). With 12 $\mathrm{Na}$ and $12 \mathrm{Cl}$ ions at the surface, the water coverage in the contact layer is estimated to be slightly less than $1 \mathrm{ML}$, where $1 \mathrm{ML}$ equals one water per surface $\mathrm{NaCl}$ pair. The highly inhomogeneous spatial probability distribution of the molecules on the surface implies a high degree of order in the first layer. The molecules are not uniformly distributed over the surface, but are "pinned" at specific adsorption sites.

Indeed, when we examine the spatial distribution of the oxygen and hydrogen atoms within the first two layers, as shown in Fig. 7(b), distorted hexagonal and squarelike hydrogen bonded rings are detected.

It is clear that the water molecules in the contact layer adopt certain preferential adsorption structures. To investigate the adsorption configurations in more detail, orientational and distance distributions of both types of adsorption structure were examined. Examples are shown in Fig. 8 in which $\mathrm{Na}-\mathrm{O}$ and $\mathrm{H}-\mathrm{Cl}$ distances between the water molecules and the substrate are plotted against $\theta$, an angle which describes the deviation of each molecule from the precise $\mathrm{Na}$ or $\mathrm{Cl}$ adsorption site (see Fig. 8 for a definition of $\theta$ ). From Fig. 8(a), we see that the most probable $\mathrm{Na}-\mathrm{O}$ distance is around $2.6 \AA$, and the preferred angle $\theta$ mainly centers around $70^{\circ}$. The $\mathrm{Na}-\mathrm{O}$ distance is a little larger than the computed $\mathrm{Na}-\mathrm{O}$ distance for a $\mathrm{Na}$ ion in liquid water, which is at 2.4-2.5 $\AA^{65,66}$ Figure 8(b) shows that the distance and angle distributions at the $\mathrm{Cl}$ site are quite different from those at the Na site. Specifically, the distribution is much broader with a wide maximum in $\mathrm{Cl}-\mathrm{H}$ distances ranging from 2.2 to $2.8 \AA$. Likewise the range of angles observed is quite broad $\left(45^{\circ}-65^{\circ}\right)$. The maximum in the $\mathrm{Cl}-\mathrm{H}$ bond length distribution at about $2.4 \AA$ is again longer than the computed $\mathrm{Cl}$ ion water distance in liquid water of $2.1 \AA^{67}$

Up to this point we have focused exclusively on the water overlayer. Now we very briefly consider the $\mathrm{NaCl}$ substrate. Specifically, we discuss how the $\mathrm{NaCl}(001)$ surface layer is altered by the presence of the water film. To this end a separate MD simulation of a clean $\mathrm{NaCl}(001)$ surface was performed at $330 \mathrm{~K}$ with an equivalent computational setup to that for the water/ $\mathrm{NaCl}$ simulation. First, we consider the interlayer spacing between the top and first subsurface layer, $d_{12}$. This is slightly expanded compared to the $0 \mathrm{~K}$ (geometry optimized) separation of $2.89 \AA$. At $330 \mathrm{~K}$, the average separation $\left\langle d_{12}\right\rangle=2.92 \AA$. When the water film is present the average separation $\left\langle d_{12}\right\rangle=2.99 \AA$. Thus the presence of the liquid water overlayer causes the interlayer spacing at $330 \mathrm{~K}$ to expand by $2.4 \%$. Considering the relative heights of the $\mathrm{Na}$ and $\mathrm{Cl}$ atoms at the surface we find, in agreement with previous DFT-PBE and experimental reports, ${ }^{29,68}$ that at the bare surface the $\mathrm{Cl}$ ions in the top layer reside $0.1 \AA$ above the $\mathrm{Na}$ ions. In the presence of the water film, the $\mathrm{Cl}$ ions remain higher than the $\mathrm{Na}$ ions but the height difference drops to $0.03 \AA$, as shown in Fig. 9. Considering the root-meansquare (rms) widths (second moments) of the ion distributions normal to the surface, we find that these are slightly increased in the presence of the water film: On the clean surface the top layer $\mathrm{Na}$ and $\mathrm{Cl} \mathrm{rms}$ widths are 0.19 and 0.20 $\AA$, respectively. In the presence of the water film the rms widths are 0.29 and $0.23 \AA$ for $\mathrm{Na}$ and $\mathrm{Cl}$, respectively. The larger fluctuations in the $\mathrm{Na}$ height are consistent with the $\mathrm{H}_{2} \mathrm{O}-\mathrm{Na}$ interaction being stronger than the $\mathrm{Cl}-\mathrm{H}_{2} \mathrm{O}$ interaction.

\section{F. Electronic properties}

We now discuss what the nature of the interaction between the water film and the $\mathrm{NaCl}$ slab is. To facilitate this, 


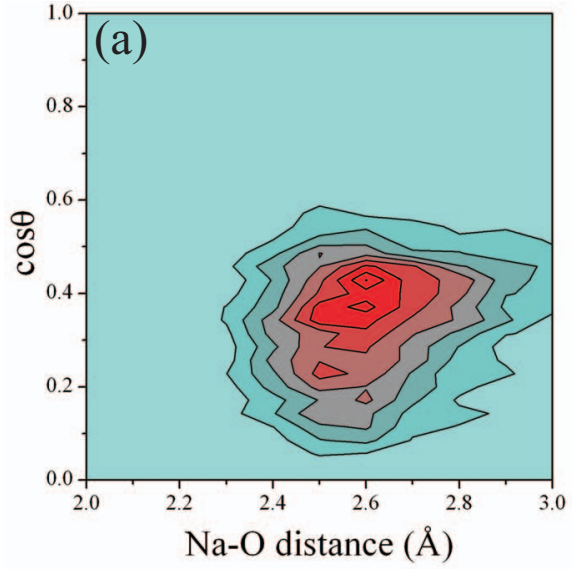

(c)

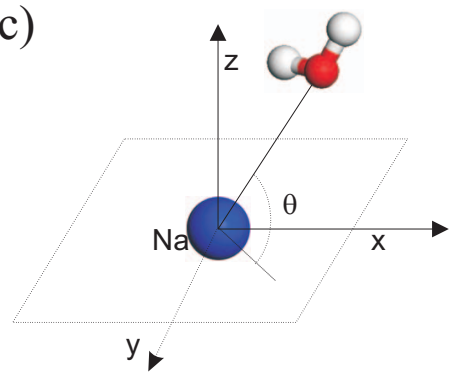

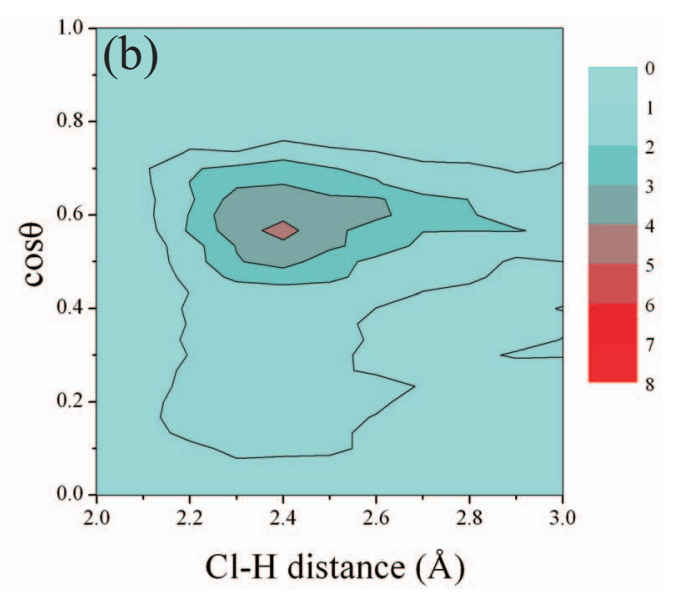

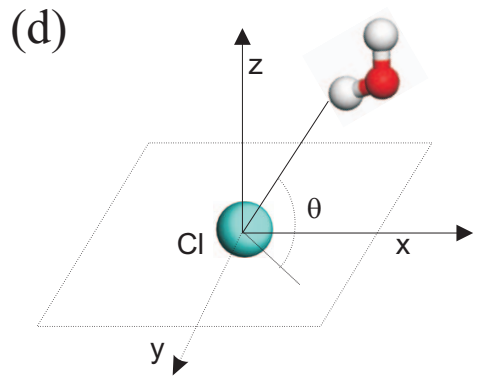

FIG. 8. (Color) Distribution of bond lengths of $\mathrm{Na}-\mathrm{O}$ (a) and $\mathrm{Cl}-\mathrm{H}(\mathrm{b})$ and the angle between the $\mathrm{Na}-\mathrm{O}$ and $\mathrm{Cl}-\mathrm{H}$ bonds and the surface $(x y)$ plane, $\theta$. Definitions of $\theta$ for the $\mathrm{Na}-\mathrm{O}$ (c) and $\mathrm{Cl}-\mathrm{H}(\mathrm{d})$ bonds. we have examined how the total electron density within the $\mathrm{NaCl}$ surface and water overlayer rearrange upon bringing them in to contact and also how the dipole moments of the water molecules in the film differ from bulk water.

First we consider the electron density rearrangement, which is defined as

$$
\Delta \rho^{e}(t)=\rho_{\mathrm{water} / \mathrm{NaCl}}^{e}(t)-\rho_{\mathrm{NaCl}}^{e}(t)-\rho_{\mathrm{water}}^{e}(t),
$$

where $\rho_{\mathrm{water} / \mathrm{NaCl}}^{e}(t)$ is the electron density of the water $/ \mathrm{NaCl}$ system at one particular time $t$ along the MD trajectory. $\rho_{\mathrm{water}}^{e}(t)$ and $\rho_{\mathrm{NaCl}}^{e}(t)$ are the electron densities of the isolated water film and the $\mathrm{NaCl}$ slab in the exact structure they adopt at time $t$ of the same trajectory. In Fig. 1(b), an example of

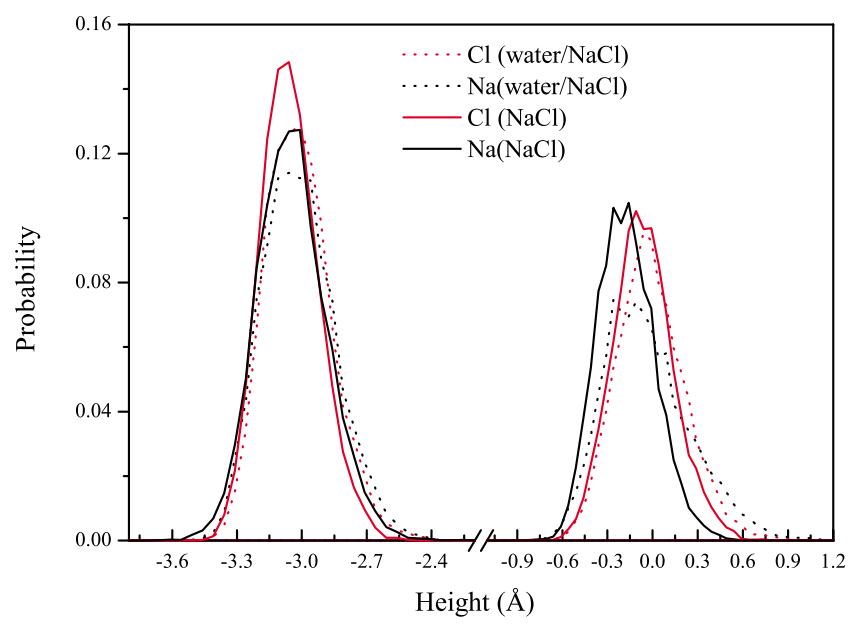

FIG. 9. (Color online) Probability distributions for the height of $\mathrm{Na}$ and $\mathrm{Cl}$ ions of the surface and subsurface layers of clean and water-covered $\mathrm{NaCl}(001)$. The zero of the $x$ axis is set to the average height of all atoms in the top layer for the water-covered surface. Each DFT-PBE simulation was performed at $330 \mathrm{~K}$.
$\Delta^{e} \rho(t)$ at one particular snapshot is displayed and in Fig. 3(d) the planar-averaged charge density difference averaged over ten equally spaced snapshots along the trajectory is displayed. The most striking conclusion of Fig. 3(d) is that the electron rearrangement experienced upon bringing the water film and $\mathrm{NaCl}$ surface into contact is limited to a very narrow region at the interface. Indeed it can be seen from Fig. 3(d) that there is negligible electron density redistribution beyond $\sim 4 \AA$ from the $\mathrm{NaCl}$ surface. Thus, the electronic influence of the surface is short ranged and molecules beyond the second layer from the surface do not "feel" the surface in any electronic sense, although, as we have discussed, structurally there is still an effect.

Now we examine the dipole moments of the individual water molecules within the film. These have been calculated from the last $1.5 \mathrm{ps}$ of the MD trajectory by means of maximally localized Wannier function centers. ${ }^{69,70}$ For reference, the average dipole moment for bulk water computed within the current computational setup is $3.11 \mathrm{D}$, which is close to other DFT-PBE results [3.10 D (Ref. 25) and 3.27 D (Ref. 51)]. It can be seen from Fig. 3(e) that in the central region (layer 4), the average dipole per water molecule is close to the bulk value: $\sim 3.09$ and $\sim 3.11 \mathrm{D}$ on average for layers 3 and 4 , respectively. At the two interfaces, however, the dipole moments of the water molecules are considerably smaller: On average $\sim 2.87 \mathrm{D}$ at the water $/ \mathrm{NaCl}$ interface (layer 1) and $\sim 2.81 \mathrm{D}$ at the water/vacuum interface (layer 5).

The reduction in the dipole moment at the two interfaces can be attributed to the reduced number of hydrogen bonds the molecules in these regions are involved in. To illustrate this we show in Fig. 10 a plot of dipole moment versus number of hydrogen bonds for the water molecules in the 


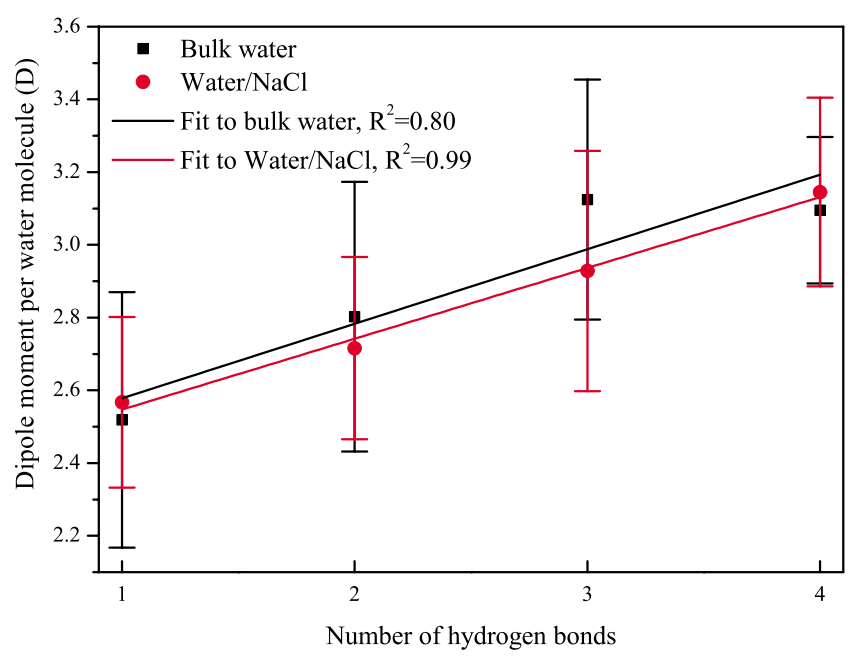

FIG. 10. (Color online) Relationship between dipole moment per water molecule and number of hydrogen bonds for bulk water and the water film on $\mathrm{NaCl}$, as obtained from a DFT-PBE simulation at $330 \mathrm{~K}$. The data points are the average values of the dipole moments and the error bars are the standard deviations. Definition I (Ref. 61) for hydrogen bonds was used.

overlayer and also in bulk water. A clear linear relationship with a correlation coefficient of 0.99 (0.80) for the water film (bulk water) is observed, revealing that as the number of hydrogen bonds increases, so too does the dipole moment. Indeed this relation can also be seen by comparing Figs. 3(c) and 3(e). Recently, McGrath et al. ${ }^{55}$ found a similar correlation for bulk water between the magnitude of the molecular dipole moment and the local tetrahedral order, although the correlation coefficient for that relation was only 0.49 .

\section{DISCUSSION}

Having presented results from a DFT-PBE simulation at $330 \mathrm{~K}$ we now briefly consider (i) how a somewhat higher temperature $(360 \mathrm{~K})$ alters the results and (ii) how the DFTPBE results compare to those obtained from a few empirical potential setups.

\section{A. Temperature}

It is now well known that DFT calculations with the PBE exchange-correlation functional predict water at room temperature that is overstructured and diffuses too slowly compared with experimental measurements. ${ }^{25,50-53,62,71,72}$ For example, Schwegler et al. ${ }^{57,73}$ obtained a diffusion coefficient of bulk water of $0.0079 \AA^{2} / \mathrm{ps}$ at $306 \mathrm{~K}$, substantially lower than the experimental values of $0.229 \AA^{2} /$ ps for $\mathrm{H}_{2} \mathrm{O}$ and $0.187 \AA^{2} / \mathrm{ps} \quad \mathrm{D}_{2} \mathrm{O}$ at $309 \mathrm{~K}^{74}$ The origin of the overstructuring and low diffusion coefficient is an important unresolved issue, widely discussed in the recent literature. ${ }^{25,50,53,57,62,63,71-73,75-78}$ For conventional PBE simulations, the overstructuring has led to the practice of simulating liquid water at slightly higher temperature than room temperature, such as the $330 \mathrm{~K}$ used here. However, the diffusion coefficient of $\mathrm{PBE}$ water at $330 \mathrm{~K}$ is still small $\left[<0.1 \AA^{2} /\right.$ ps (Ref. 50)]. For the water film on $\mathrm{NaCl}$ computed here the diffusion coefficient is also $0.1 \AA^{2} / \mathrm{ps}$. In order to understand how temperature will affect the density profile for water on $\mathrm{NaCl}$, another MD simulation at $360 \mathrm{~K}$

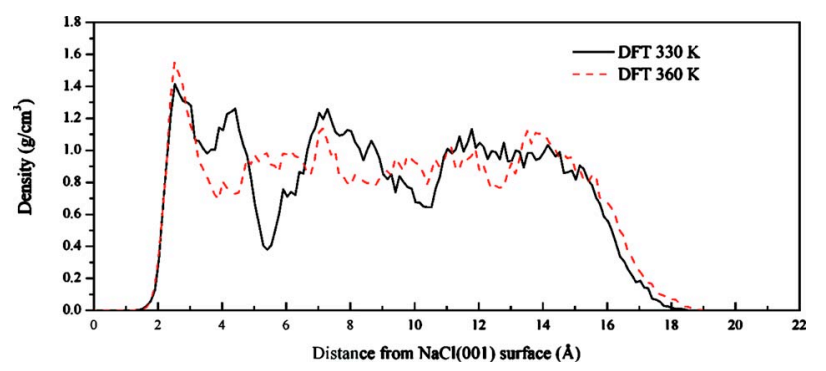

FIG. 11. (Color online) Planar-averaged water densities along the $z$ axis calculated by DFT-PBE at 330 and $360 \mathrm{~K}$ for water/ $\mathrm{NaCl}(001)$. The profile at $330 \mathrm{~K}$ is the same as the one displayed in Fig. 3(a).

was performed. Apart from the higher temperature all other aspects of this simulation were the same as the one at $330 \mathrm{~K}$. The diffusion coefficient obtained for the molecules in the water film at $360 \mathrm{~K}$ is $0.2 \AA^{2} / \mathrm{ps}$, twice that obtained at 330 K. In Fig. 11, the water density profiles perpendicular to the surface from the 360 and $330 \mathrm{~K}$ simulations are compared. The comparison between the two temperatures reveals that close to the surface, the large planar-averaged density enhancement is still apparent, in fact, the first peak maximum increases slightly from 1.40 to $1.55 \mathrm{~g} / \mathrm{cm}^{3}$. However, beyond the immediate interfacial zone the planar-averaged density oscillations are significantly reduced in the higher temperature simulation. Thus the higher temperature mainly affects the water density away from water/ $\mathrm{NaCl}$ interface and only has a minor impact on the water density close the water/ $\mathrm{NaCl}$ interface.

\section{B. Empirical potential simulations}

We now briefly consider how the present $a b$ initio MD results compare to those obtained with a few popular force field setups. We mentioned in Sec. I that large enhancements in the planar-averaged water density at the $\mathrm{NaCl}$ interface have been reported before; density maxima of up to $\sim 3.4 \mathrm{~g} / \mathrm{cm}^{3}$. ${ }^{16}$ We do indeed obtain a large value for the density maximum from our ab initio simulations. However, it is on the order of a $40 \%\left(1.4 \mathrm{~g} / \mathrm{cm}^{3}\right.$, Fig. 3) increase and certainly not a $240 \%$ increase. This prompted us to compare our DFT results to those obtained with empirical potentials for the precise system examined here, i.e., a nanometer thick water film on a flat $\mathrm{NaCl}(001)$ surface. Two water models, SPC/E (Ref. 79) and TIP3P, ${ }^{48}$ were used along with a range of typical water-ion parameters used in previous studies, ${ }^{49,80-82}$ as listed in Table III. Each force field simulation was run for at least 200 ps at $300 \mathrm{~K}$ with the last 20 ps used for analysis purposes.

The calculated planar-averaged density profiles obtained with the various force field setups are shown in Fig. 12. Focusing on the height of the planar-averaged density maximum, it can be seen that this is very sensitive to the choice of water-ion interaction and that all options considered yield a considerably larger density maximum than that obtained from DFT. Whereas the first peak maximum from DFT is $1.4 \mathrm{~g} / \mathrm{cm}^{3}$, with the force field models it ranges from $\sim 2$ to $\sim 3.2 \mathrm{~g} / \mathrm{cm}^{3}$. The choice of the water-ion interaction is crucial, with the density profile being less sensitive to the choice of water model (for the two tested). Of the water-ion param- 


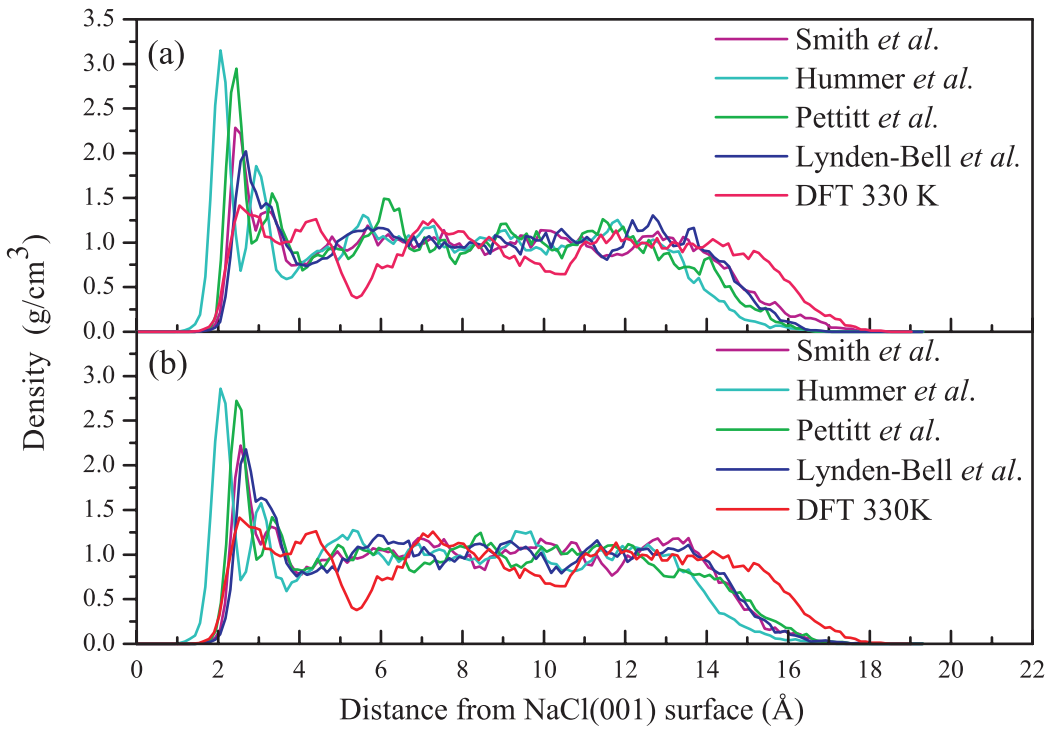

FIG. 12. (Color) Planar-averaged water densities along the $z$ axis calculated using different water-ion parameters for water $\mathrm{NaCl}(001)$, compared with DFT (330 $\mathrm{K})$ : (a) is with TIP3P water; (b) is with SPC/E water. The water-ion parameters used in these simulations and attributed to the various authors in the legend are given in Table III.

eters tested, the parameters of Hummer et al. ${ }^{80}$ give the largest first peak maximum: $3.17(2.86) \mathrm{g} / \mathrm{cm}^{3}$ with TIP3P (SPC/E) water. The parameters of Smith et al. ${ }^{49}$ and LyndenBell et $a .^{81}$ yield the smallest peaks: 2.28(2.22) and $2.02(2.17) \mathrm{g} / \mathrm{cm}^{3}$ using TIP3P (SPC/E) water, respectively. Moreover, the parameters of Pettitt et al. ${ }^{82}$ yield a density maximum of 3.00(2.72) $\mathrm{g} / \mathrm{cm}^{3}$ using TIP3P (SPC/E).

To understand why the various parameters resulted in such large differences in the density maximum, we calculated the adsorption energy of $\mathrm{a}_{2} \mathrm{O}$ monomer on the $\mathrm{NaCl}$ surface using TIP3P and SPC/E, with the four different sets of $\mathrm{H}_{2} \mathrm{O}$-ion parameters. The water monomer was adsorbed above a $\mathrm{Na}$ site, which is predicted by DFT to be the most stable site for water monomer adsorption. ${ }^{26-29}$ As shown in Fig. 13, a correlation between the water adsorption energy and the first peak maximum of the water density is observed. Specifically, the larger the adsorption energy, the larger the buildup in the water density at the $\mathrm{NaCl}$ interface.

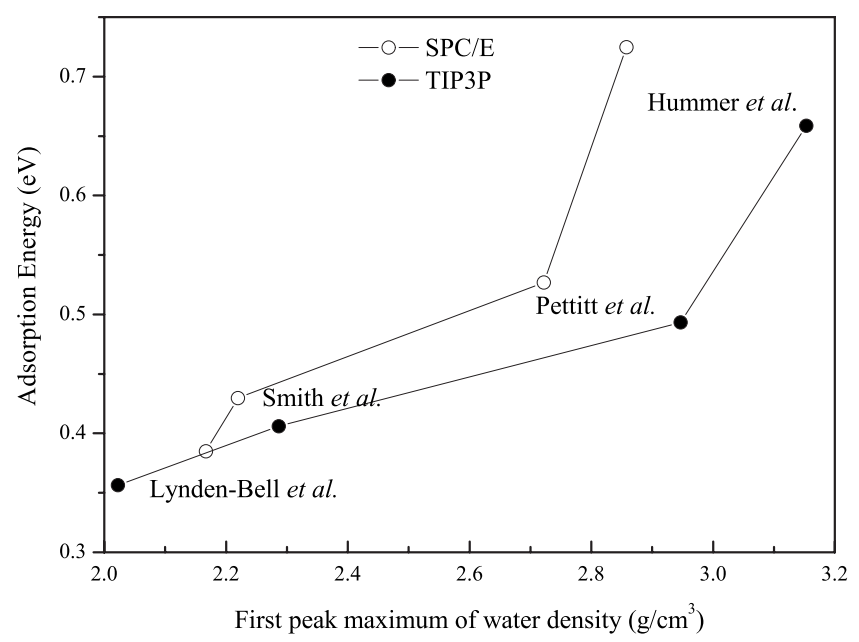

FIG. 13. Relationship between the height of the first peak in the planaraveraged density for water at $\mathrm{NaCl}$ and the adsorption energy obtained with the different water-ion parameters given in Table III. The filled circles correspond to SPC/E and the unfilled circles to TIP3P water.

\section{SUMMARY AND CONCLUSIONS}

In conclusion, $a b$ initio MD simulations of a liquid water-salt interface have been performed. The model was chosen to resemble the thin nanoscale water layer that forms on the $\mathrm{NaCl}(100)$ prior to deliquescence. The simulations demonstrate that the interaction with the substrate is sufficient to pin the water molecules in the contact layer into specific adsorption configurations. The interaction with the substrate also leads to a buildup in water density in the immediate vicinity of the substrate, which produces damped oscillations in the planar-averaged density along the surface normal. Once one is about $8 \AA$ from the substrate, the bulk liquid water density is recovered and all other properties of the water film considered resemble those of bulk PBE liquid water. A large proportion of broken hydrogen bonds is observed at the water/ $\mathrm{NaCl}$ and water/vacuum interfaces, leading in part to the observation of a linear correlation between the number of hydrogen bonds a molecule is involved and its dipole moment. Should one wish to perform accurate simulations of the liquid water $/ \mathrm{NaCl}$ interface with empirical potentials, then we suggest that such potentials should also be capable of capturing the reduction in dipole moment at the water $/ \mathrm{NaCl}$ interface and, in more general terms, the reduction in dipole moments at the water/vacuum interface too. Here we tested a couple of common nonpolarizable potentials (TIP3P and SPC/E) and found that they predicted planar-averaged density enhancements in the immediate vicinity of the contact layer that were much larger than that predicted by PBE. In ongoing work we are examining this system with alternative empirical potential schemes. In light of our recent high precision determination of the adsorption energy of a water monomer on $\mathrm{NaCl}$ of $\sim 0.5 \mathrm{eV},{ }^{64}$ about 50 meV larger than the PBE value obtained here, we are also exploring this system with alternative exchange-correlation functionals and attempting to ascertain the importance of van der Waals (dispersion) forces for water on salt.

\section{ACKNOWLEDGMENTS}

This work was supported through the EURYI award scheme (see www.esf.org/euryi) and the EPSRC. Computa- 
tional resources from the London Centre for Nanotechnology are greatly appreciated. Also via our membership of the U.K.'s HPC Materials Chemistry Consortium, which is funded by EPSRC-GB (Grant No. EP/F067496), this work made use of the facilities of HECToR, the U.K.'s national high-performance computing service, which is provided by UoE HPCx Ltd at the University of Edinburgh, Cray Inc. and NAG Ltd, and funded by the Office of Science and Technology through EPSRC's High End Computing Programme.

${ }^{1}$ S. J. Peters and G. E. Ewing, J. Phys. Chem. B 101, 10880 (1997).

${ }^{2}$ G. E. Ewing, Chem. Rev. (Washington, D.C.) 106, 1511 (2006).

${ }^{3}$ S. Fölsch, A. Stock, and M. Henzler, Surf. Sci. 264, 65 (1992).

${ }^{4}$ L. W. Bruch, A. Glebov, J. P. Toennies, and H. Weiss, J. Chem. Phys. 103, 5109 (1995).

${ }^{5}$ J. P. Toennies, F. Traeger, J. Vogt, and H. Weiss, J. Chem. Phys. 120, 11347 (2004).

${ }^{6}$ J. M. Park, J. H. Cho, and K. S. Kim, Phys. Rev. B 69, 233403 (2004).

${ }^{7}$ P. Cabrera-Sanfelix, A. Arnau, G. R. Darling, and D. Sanchez-Portal, J. Chem. Phys. 126, 214707 (2007).

${ }^{8}$ B. J. Finlayson-Pitts, Chem. Rev. (Washington, D.C.) 103, 4801 (2003).

${ }^{9}$ A. Verdaguer, G. M. Sacha, H. Bluhm, and M. Salmeron, Chem. Rev. (Washington, D.C.) 106, 1478 (2006).

${ }^{10}$ M. Luna, F. Rieutord, N. A. Melman, Q. Dai, and M. Salmeron, J. Phys. Chem. A 102, 6793 (1998)

${ }^{11}$ A. Verdaguer, G. M. Sacha, M. Luna, D. F. Ogletree, and M. Salmeron, J. Chem. Phys. 123, 124703 (2005).

${ }^{12}$ K. Ichikawa, S. Sato, and N. Shimomura, Pure Appl. Chem. 76, 115 (2004).

${ }^{13}$ J. Arsic, D. M. Kaminski, N. Radenovic, P. Poodt, W. S. Graswinckel, H. M. Cuppen, and E. Vlieg, J. Chem. Phys. 120, 9720 (2004).

${ }^{14}$ N. Anastasiou, D. Fincham, and K. Singer, J. Chem. Soc., Faraday Trans. 79, 1639 (1983)

${ }^{15}$ R. Bahadur, L. M. Russell, S. Alavi, S. T. Martin, and P. R. Buseck, J. Chem. Phys. 124, 154713 (2006)

${ }^{16}$ H. Shinto, T. Sakakibara, and K. Higashitani, J. Phys. Chem. B 102, 1974 (1998)

${ }^{17}$ E. Stöckelmann and R. Hentschke, J. Chem. Phys. 110, 12097 (1999).

${ }^{18}$ C. Y. Lee, J. A. McCammon, and P. J. Rossky, J. Chem. Phys. 80, 4448 (1984).

${ }^{19}$ J. Martí, G. Nagy, E. Guàrdia, and M. C. Gordillo, J. Phys. Chem. B 110 , 23987 (2006).

${ }^{20}$ J. Arsic, D. Kaminski, P. Poodt, and E. Vlieg, Phys. Rev. B 69, 245406 (2004).

${ }^{21}$ J. G. Catalano, C. Park, Z. Zhang, and P. Fenter, Langmuir 22, 4668 (2006).

${ }^{22}$ S. H. Lee and P. J. Rossky, J. Chem. Phys. 100, 3334 (1994).

${ }^{23}$ E. Johnson, Science 296, 477 (2002).

${ }^{24}$ L. Cheng, P. Fenter, K. L. Nagy, M. L. Schlegel, and N. C. Sturchio, Phys. Rev. Lett. 87, 156103 (2001).

${ }^{25}$ G. Cicero, J. C. Grossman, E. Schwegler, F. Gygi, and G. Galli, J. Am. Chem. Soc. 130, 1871 (2008).

${ }^{26}$ H. Meyer, P. Entel, and J. Hafner, Surf. Sci. 488, 177 (2001).

${ }^{27}$ Y. Yang, S. Meng, and E. G. Wang, Phys. Rev. B 74, 245409 (2006).

${ }^{28}$ P. Cabrera-Sanfelix, A. Arnau, G. R. Darling, and D. Sanchez-Portal, J. Phys. Chem. B 110, 24559 (2006).

${ }^{29}$ B. Li, A. Michaelides, and M. Scheffler, Phys. Rev. B 76, 075401 (2007).

${ }^{30}$ G. Cicero, J. C. Grossman, A. Catellani, and G. Galli, J. Am. Chem. Soc. 127, 6830 (2005).

${ }^{31}$ K. N. Kudin and R. Car, J. Am. Chem. Soc. 130, 3915 (2008).

${ }^{32}$ F. Schiffmann, J. Hutter, and J. VandeVondele, J. Phys.: Condens. Matter 20, 064206 (2008).

${ }^{33}$ P. Raybaud, M. Digne, R. Iftimie, W. Wellens, P. Euzen, and H. Toulhoat, J. Catal. 201, 236 (2001).

${ }^{34}$ R. N. Barnett and U. Landman, J. Phys. Chem. 100, 13950 (1996).

${ }^{35}$ T. Asada and K. Nishimoto, Chem. Phys. Lett. 232, 518 (1995).

${ }^{36}$ P. Jungwirth, J. Phys. Chem. A 104, 145 (2000).

${ }^{37}$ S. Yamabe, H. Kouno, and K. Matsumura, J. Phys. Chem. B 104, 10242 (2000).

${ }^{38}$ A. Pramanik, R. P. Kalagi, V. J. Barge, and S. R. Gadre, Theor. Chem. Acc. 114, 129 (2005)
${ }^{39}$ S. S. M. C. Godinho, P. C. do Couto, and B. J. C. Cabral, Chem. Phys. Lett. 399, 200 (2004).

${ }^{40}$ C. K. Siu, B. S. Fox-Beyer, M. K. Beyer, and V. E. Bondybey, Chem.Eur. J. 12, 6382 (2006).

${ }^{41}$ L. Liu, M. Krack, and A. Michaelides, J. Am. Chem. Soc. 130, 8572 (2008).

${ }^{42}$ J. VandeVondele, M. Krack, F. Mohamed, M. Parrinello, T. Chassaing, and J. Hutter, Comput. Phys. Commun. 167, 103 (2005).

${ }^{43}$ J. P. Perdew, K. Burke, and M. Ernzerhof, Phys. Rev. Lett. 77, 3865 (1996); 78, 1396 (1997).

${ }^{44}$ S. Goedecker, M. Teter, and J. Hutter, Phys. Rev. B 54, 1703 (1996); C. Hartwigsen, S. Goedecker, and J. Hutter, ibid. 58, 3641 (1998); M. Krack, Theor. Chem. Acc. 114, 145 (2005).

${ }^{45}$ S. G. Louie, S. Froyen, and M. L. Cohen, Phys. Rev. B 26, 1738 (1982).

${ }^{46}$ Handbook of Chemistry and Physics, 76th ed. (CRC, Boca Raton, 1995).

${ }^{47}$ F. G. Fumi and M. P. Tosi, J. Phys. Chem. Solids 25, 31 (1964); M. P. Tosi and F. G. Fumi, ibid. 25, 45 (1964).

${ }^{48}$ W. L. Jorgensen, J. Chandrasekhar, J. D. Madura, R. W. Impey, and M. L. Klein, J. Chem. Phys. 79, 926 (1983).

${ }^{49}$ D. E. Smith and L. X. Dang, J. Chem. Phys. 100, 3757 (1994).

${ }^{50}$ J. VandeVondele, F. Mohamed, M. Krack, J. Hutter, M. Sprik, and M. Parrinello, J. Chem. Phys. 122, 014515 (2005).

${ }^{51}$ T. Todorova, A. P. Seitsonen, J. Hutter, I. F. W. Kuo, and C. J. Mundy, J. Phys. Chem. B 110, 3685 (2006).

${ }^{52}$ M. Sharma, R. Resta, and R. Car, Phys. Rev. Lett. 98, 247401 (2007).

${ }^{53}$ J. A. Morrone and R. Car, Phys. Rev. Lett. 101, 017801 (2008).

${ }^{54}$ M. J. McGrath, J. I. Siepmann, I. F. W. Kuo, C. J. Mundy, J. VandeVondele, J. Hutter, F. Mohamed, and M. Krack, ChemPhysChem 6, 1894 (2005).

${ }^{55}$ M. J. McGrath, J. I. Siepmann, I. F. W. Kuo, and C. J. Mundy, Mol. Phys. 104, 3619 (2006).

${ }^{56}$ I. F. W. Kuo and C. J. Mundy, Science 303, 658 (2004).

${ }^{57}$ E. Schwegler, J. C. Grossman, F. Gygi, and G. Galli, J. Chem. Phys. 121, 5400 (2004).

${ }^{58}$ A. Luzar and D. Chandler, Nature (London) 379, 55 (1996).

${ }^{59}$ A. Luzar, J. Chem. Phys. 113, 10663 (2000).

${ }^{60} \mathrm{Ph}$. Wernet, D. Nordlund, U. Bergmann, M. Cavalleri, M. Odelius, H. Ogasawara, L. Å. Näslund, T. K. Hirsch, L. Ojamäe, P. Glatzel, L. G. M. Pettersson, and A. Nilsson, Science 304, 995 (2004).

${ }^{61}$ The following five definitions of hydrogen bonds are used. (I) The distance, $\mathrm{R}_{\mathrm{OD}-\mathrm{OA}}$, between donor and acceptor oxygen atoms is $<3.5 \AA$ and the angle, $\beta=\angle \mathrm{H}_{\mathrm{D}}-\mathrm{O}_{\mathrm{D}}-\mathrm{O}_{\mathrm{A}}$, between the $\mathrm{H}_{\mathrm{D}}-\mathrm{O}_{\mathrm{D}}$ bond and the $\mathrm{O}_{\mathrm{D}}-\mathrm{O}_{\mathrm{A}}$ vectors is $<30^{\circ}$ (Ref. 58). Here, $\mathrm{H}_{\mathrm{D}}$ and $\mathrm{O}_{\mathrm{D}}$ represent the donor hydrogen and oxygen atom, and $\mathrm{H}_{\mathrm{A}}$ and $\mathrm{O}_{\mathrm{A}}$ represent the acceptor hydrogen and oxygen, respectively. (II) The $\mathrm{R}_{\mathrm{OD}-\mathrm{OA}}$ distance is $<3.5 \AA$ and the angle, $\alpha=\angle \mathrm{H}_{\mathrm{D}}-\mathrm{O}_{\mathrm{A}}-\mathrm{H}_{\mathrm{A}}$, is $>135^{\circ}$ (Ref. 51). (III) The $\mathrm{R}_{\mathrm{OD}-\mathrm{HA}}$ bond must be $\leq 2.27 \AA$ and the angle, $\gamma=\angle \mathrm{H}_{\mathrm{D}}-\mathrm{O}_{\mathrm{D}}-\mathrm{O}_{\mathrm{A}}$, is $\leq 30^{\circ}$ (Ref. 59); (IV) The $\mathrm{R}_{\mathrm{OD}-\mathrm{HA}}$ bond is between $1.59 \AA$ and $2.27 \AA$ and the angle, $\alpha$ $=\angle \mathrm{H}_{\mathrm{D}}-\mathrm{O}_{\mathrm{A}}-\mathrm{H}_{\mathrm{A}}$, is $>140^{\circ}$ (Ref. 56). (V) The distance $\mathrm{R}_{\mathrm{OD}-\mathrm{OA}}$ $\leq-0.00044 \beta^{2}+3.3 \AA$ (Ref. 60).

${ }^{62}$ M. V. Fernández-Serra and E. Artacho, J. Chem. Phys. 121, 11136 (2004).

${ }^{63}$ D. Prendergast and G. Galli, Phys. Rev. Lett. 96, 215502 (2006).

${ }^{64}$ B. Li, A. Michaelides, and M. Scheffler, Surf. Sci. 602, L135 (2008).

${ }^{65}$ J. A. White, E. Schwegler, G. Galli, and F. Gygi, J. Chem. Phys. 113, 4668 (2000).

${ }^{66}$ T. Ikeda, M. Boero, and K. Terakura, J. Chem. Phys. 126, 034501 (2007).

${ }^{67}$ J. M. Heuft and E. J. Meijer, J. Chem. Phys. 119, 11788 (2003).

${ }^{68}$ J. G. Roberts, S. Hoffer, M. A. Van Hove, and G. A. Somorjai, Surf. Sci. 437, 75 (1999).

${ }^{69}$ N. Marzari and D. Vanderbilt, Phys. Rev. B 56, 12847 (1997).

${ }^{70}$ P. L. Silvestrelli and M. Parrinello, Phys. Rev. Lett. 82, 3308 (1999).

${ }^{71}$ Y. A. Mantz, B. Chen, and G. J. Martyna, J. Phys. Chem. B 110, 3540 (2006)

${ }^{72}$ H. S. Lee and M. E. Tuckerman, J. Chem. Phys. 126, 164501 (2007).

${ }^{73}$ J. C. Grossman, E. Schwegler, E. W. Draeger, F. Gygi, and G. Galli, J. Chem. Phys. 120, 300 (2004).

${ }^{74}$ R. Mills, J. Phys. Chem. 77, 685 (1973).

${ }^{75}$ M. Sharma, R. Resta, and R. Car, Phys. Rev. Lett. 95, 187401 (2005).

${ }^{76}$ B. Santra, A. Michaelides, and M. Scheffler, J. Chem. Phys. 127, 184104 (2007).

${ }^{77}$ B. Santra, A. Michaelides, M. Fuchs, A. Tkatchenko, C. Filippi, and M. 
Scheffler, J. Chem. Phys. 129, 194111 (2008).

${ }^{78}$ T. Head-Gordon and M. E. Johnson, Proc. Natl. Acad. Sci. U.S.A. 103, 7973 (2006)

${ }^{79}$ P. Mark and L. Nilsson, J. Phys. Chem. A 105, 9954 (2001).
${ }^{80}$ G. Hummer, L. R. Pratt, and A. E. Garcia, J. Phys. Chem. 100, 1206 (1996).

${ }^{81}$ R. M. Lynden-Bell and J. C. Rasaiah, J. Chem. Phys. 107, 1981 (1997).

${ }^{82}$ B. M. Pettitt and P. J. Rossky, J. Chem. Phys. 84, 5836 (1986). 